\title{
Implementation of Corrosion Prevention Practices for Ground Vehicles
}

\author{
Xuecheng Zhang ${ }^{*}$, Qixun Liu and Lu Jiang \\ Military Representative Office in Shanghai; Shanghai, 201109, China \\ xc-zhang@outlook.com
}

Keywords: Ground vehicle, Corrosion prevention, Material selection, Protective coatings, Regular maintenance

\begin{abstract}
Ground vehicles are operated in severe environments at the risk of corrosion. The corrosion would degrade military readiness and cause a large amount of cost. This paper describes some engineering practices of corrosion prevention for ground vehicles based on results of the environmental test and field operation from previous products and similar ones. The influence of environmental factors on the corrosion of ground vehicles is analyzed. And then some corrosion control methods proven by engineering practices are introduced. These practices could enhance the operational readiness and reduce unquantifiable lifecycle maintenance costs of ground vehicles.
\end{abstract}

\section{Introduction}

For military missions, ground vehicles are deployed and operated in various environmental zones. Environmental elements acting on vehicles vary in sun exposure, humidity, mould, dust, temperature and pressure. Corrosion would occur naturally when ground vehicles are exposed to these elements. The phenomenon of corrosion is defined as the unintended destruction or deterioration of a material or its properties because of a chemical or electrochemical reaction with its environments [1]. Corrosion has a negative effect on the ground vehicle's mission capability and causes a large amount of cost. The failure of key components would cause partial or even full loss of fighting capacity. According to a 2010 study directed by U.S. Department of Defense, corrosion cost for army ground vehicles takes up about 25 percent of the total field-level maintenance costs [2]. The corrosion cost of the high mobility multi-purpose wheeled vehicle was reported about to be \$2.0-2.5 billion per year [3]. Corrosion is a problem because it degrades the readiness and shortens the use time of ground vehicles.

Methods and regulations of corrosion prevention and control are adopted by design engineers to ensuring the sustained performance and readiness of ground vehicles. However, the environmental applicability of ground vehicles is still a challenge because it should be applied in a wide range of operational conditions. The ground vehicle is an outdoor platform used in all weather conditions. Therefore it is frequently operated for a long time in the bad weather, such as rain-snow, sandstorm, wet and salt-fog. These environmental factors would seriously intensify the behavior of corrosion. Consequently, readiness of the ground vehicle is adversely affected. Corresponding protection measures should be taken to make ground vehicles working normally in diverse conditions considering special requirements from both designing aspects and vehicle's usage. Some engineering practices of corrosion prevention for ground vehicles are described in this paper based on results of the environmental test and field operation from previous products and similar ones.

\section{Atmospheric Corrosion Mechanism}

Corrosion of materials and products goes together with different environmental factors and their intensities of operational conditions. The phenomenon would be very complex by the combined action of multiple factors in various environments. And different materials have their own corrosion pattern or environmental adaptability.

Corrosion of metal and alloys. The ground vehicle is mainly made up from metallic components, which are essentially corrosive materials. The moisture in the atmosphere condenses to form a thin 
water film on the component surface. Then corrosion results from the electro-chemical reaction between the metallic molecule and the water film.

Metal corrosion. According to Eq.1, the metal is dissolved as hydration ion leaving equivalent electrons $\left(\mathrm{e}^{-}\right)$at the anode. And the adsorbed species $\mathrm{OH}^{-}$is formed through oxidizing depolarization process at the cathode, shown as Eq.2, which accelerates the transition of metal from $\mathrm{M}$ to $\mathrm{M}^{n+}$. The corrosion speed would increase rapidly when the wetness of the exposed atmosphere exceeds a critical value, called critical humidity. The range of critical humidity for most metals is $60 \% \sim 70 \%$, whose magnitude would decrease when pollutants, such as $\mathrm{CO}_{2}$ and compound of salt, are contained in the atmosphere.

$$
\begin{aligned}
& \mathrm{M} \rightarrow \mathrm{M}^{n+}+n \mathrm{e}^{-} \\
& \mathrm{H}_{2} \mathrm{O}+1 / 2 \mathrm{O}_{2}+2 \mathrm{e}^{-} \rightarrow 2 \mathrm{OH}^{-}
\end{aligned}
$$

The corrosion in operational conditions is influenced by the combination of multiple factors, which include not only relative humidity $(\mathrm{RH})$, rain and temperature, but also pollutants such as sulfur ions $\left(\mathrm{SO}_{2}\right)$ and chloride ions $\left(\mathrm{C1}^{-}\right)$. The outdoor atmospheric corrosion rate model proposed by Mendoza and Corvo [4] is shown in Eq.3, which reflects the influence of the relationship between the wetness and main pollutants' deposition rates $\left(V_{\mathrm{cl}^{-}}\right.$and $\left.V_{\mathrm{SO}_{2}}\right)$. The corrosion of metallic materials is a function of the severity of environmental factors and time exposed to environmental conditions. To reflect the evaporation of the electrolyte layer at high temperature, this model divides the usual time of wetness ( $\mathrm{RH} 80 \%-100 \%)$ into two parts of the time of wetness at temperatures of $5-25^{\circ} \mathrm{C}\left(\tau_{5-25}\right)$ and $25-35^{\circ} \mathrm{C} \quad\left(\tau_{25-35}\right)$.

$$
\begin{aligned}
C= & a+b V_{\mathrm{cl}^{-}} \tau_{5-25}+c V_{\mathrm{cl}^{-}} \tau_{25-35}+d V_{\mathrm{SO}_{2}} \tau_{5-25}+e V_{\mathrm{SO}_{2}} \tau_{25-35}+f V_{\mathrm{cl}^{-}} t_{\text {rain }} \\
& +g V_{\mathrm{SO}_{2}} t_{\text {rain }}+h r_{f} / t_{\text {rain }}
\end{aligned}
$$

where $C$ is the weight loss in $\mathrm{g} / \mathrm{m}^{2}$ at periods of 6,12 and 18 months of exposure; $t_{\text {rain }}$ and $r_{f}$ are the time and magnitude of rainfall, respectively.

Alloy corrosion. Alloys have better corrosion resistance because there is a passivation film on the surface. The stainless steel is a typical alloy with economical and extensive application. The stainless steel would arise more or less corrosion with pitting from scratches on the surface. Alloy elements influence significantly the corrosion resistance of stainless steel. Table 1 shows experiment results of four-year average corrosion rate of several stainless steel samples exposed in the coastal atmosphere.

Table1 Average corrosion rate of different stainless steels in coastal atmosphere

\begin{tabular}{|c|c|c|c|c|}
\hline & $\mathrm{S} 42000$ & $\mathrm{~S} 30400$ & $\mathrm{~S} 32100$ & $\mathrm{~S} 31603$ \\
\hline corrosion rate $[\mu \mathrm{m} / \mathrm{a}]$ & 3.50 & 0.38 & 0.36 & 0.15 \\
\hline
\end{tabular}

The coastal atmosphere is much more corrosive due to damp-heat and strong concentrations of chloride ions. To a certain extent, the situation of metallic materials in the coastal atmosphere reveals the degree of product's environmental adaptability.

Corrosion of coatings. The surface of metallic components is usually clad or treated in order to prevent or mitigate corrosion except for special requirements. Metal plating and organic coating are mainly two kinds of coatings.

Metal plating. The metal plating protects base metal from two aspects: one is to prevent the base metal's corrosion by the mask of compact oxide on the surface; the other is to be used as the opposite polarity of electro-chemical reaction when the surface plating is damaged. Table 2 shows experiment results of the service life of different zinc plating thicknesses in diverse atmospheres. Therefore corrosion prevention is related to the plating thickness and operational conditions. Experience discovers the thickness of metal plating should be more than $5 \mu \mathrm{m}$ used in the outdoor condition and above $25 \mu \mathrm{m}$ to meet the requirement of ten-year service life in the severe corrosion atmosphere.

Table 2 Service life of different zinc plating thicknesses [year/a]

\begin{tabular}{|c|c|c|c|c|c|}
\hline thickness $[\mu \mathrm{m}]$ & 7.0 & 14.0 & 19.3 & 24.5 & 31.5 \\
\hline in rural atmosphere & 4.5 & 10.5 & 15.5 & 20.8 & 28.3 \\
\hline in coastal atmosphere & 2.0 & 4.4 & 6.4 & 8.5 & 11.3 \\
\hline
\end{tabular}


Organic coatings. They are mainly influenced by solar radiation and pollutants $\left(\mathrm{C}^{-}{ }^{-}\right.$and $\left.\mathrm{SO}_{2}\right)$. The failure model of organic coatings primarily includes blistering, powdering, rusting, and cracking, et al. The eight-year experiment results of different coatings exposed in various conditions prove that corrosion rates are high especially in the plateau and coastal atmospheres [5].

Corrosion of rubber materials. The corrosion characteristics of rubber materials are similar to those of organic coatings. Rubbers for sealing also undergo stress resulting in fatigue and aging. This would lead to the loss of elasticity and shock absorption.

\section{Corrosion Prevention Measures}

Various parts of the ground vehicle undergo different environmental influences due to the complex structure and framework. Therefore targeted protection measures should be taken considering the location of parts in various environmental conditions. Studies prove that proper investment in prevention and mitigation of corrosion during design, manufacture, and sustainment could decrease 30 percent of corrosion costs [6]. To improve the corrosion resistance of the ground vehicle, some measures are taken which include material selection, such as corrosion-resistant alloys, plastics, and polymers; organic and metallic protective coatings; suitable technique and appropriate maintenance.

Protection of bare metallic parts. The term "bare" means the surface of metallic parts has no coating. These parts commonly include carbon steel standard parts; stainless nut and pipeline; aluminium alloy plug; cable connector; and mounting bracket, et al.

Carbon steel. These parts such as bolt, nut and screw, are replaced with stainless steel ones. They are coated if carbon steel is needed for special requirements.

Stainless steel. If the tensile strength could satisfy demand, S31603 stainless steel is selected preferentially. Part surface is passivated first and then coated. Furthermore, it is insulated from other materials to avoid direct contact. Sealant is used to seal the junction between bolt and nut.

Aluminum. Antirust aluminiums or forging aluminums are selected if aluminium needs to be used as the substrate. To get better performance, antirust aluminiums are first processed by the sulfur acid anodizing or conduction oxidation and then coated.

In addition, some protective techniques are adopted to improve the corrosion resistance. Fastening parts for aluminium shelter, such as bolt, nut and rivet, are fitted with sealant. For example, corrosion inhibitors or anti-rust primers are sprayed to conduct wet assembly in order to avoid galvanic corrosion of the connection between steel or stainless steel and aluminium.

Protection of rubbers and plastics. There are many uncovered parts made from rubbers and plastics, such as rubber strip on the window, sealing ring, and rubber pad. These parts are easy to fail exposed to solar radiation and high temperature for a long time. Fatigues would cause the leakage of oil/gas or short-circuit of cables resulting in severe consequences. Therefore, rubber materials with good aging resistance performance, such as silicone rubber and acrylate rubber, are recommended to be used for cable sheaths and sealing parts. Besides, some parts are taken as vulnerable parts with more spare parts if they could not be replaced with better performance materials.

Protection of coatings. Coatings are very important for performance and the appearance condition. Coatings could effectively mitigate the corrosion of metallic parts like steel and aluminium. They prevent the substrate from severe corrosion ensuring the normal structure function. The iron red epoxy ester is selected as the primer of coatings for ferrous metals and zinc yellow epoxy ester for nonferrous metals according to experiments and field practices. The acrylic polyurethane semi-gloss enamel is selected as the topcoat. Engineering practices prove that the thickness of coatings ranges between $90 \mu \mathrm{m}$ and $150 \mu \mathrm{m}$ for equipments outside while $80 \mu \mathrm{m}$ and $140 \mu \mathrm{m}$ for those in board.

Attention should be paid to some special parts such as engine exhaust. The working temperature of engine exhaust is very high. Subject to alternate heat and cold, it suffers much more severe erosion by the concentrated humidity and chemical corrosive medium. Experience has verified the two moves against corrosion effective: one is to add damp-proof primer, such as epoxy or phenolic aldehyde paint, considering the compatibility with the heat resistant aluminium paint; the other is to increase 
painting times or coating's thickness in order to reduce coating gap defect and increase coverage rate of the paint film. This could restrain the permeation of the electrolyte.

Regular corrosion control maintenance. The appropriate maintenance is very important to prevent corrosion. The maintenance cycle and items should be determined according to the operational conditions. Maintenance cycle is about 3 months in the plateau atmosphere and 2 months in the coastal atmosphere. The paint should be repaired if coatings have failures of blistering, cracking or peeling. The bare metallic parts should be protected in time if rust is obvious on the surface. Nonmetallic parts should be repaired or replaced when their functions diminish evidently or exterior shows distinct signs of deterioration. Some parts should be checked carefully due to special structures or the hostile working environment.

Connection or edge. These positions like screws' connection and the weld's edge would become weak points if the paint film is scratched/knocked or sprayed with non-uniform thickness. Therefore action of clssing should be taken when rust or breakages happen.

Parts in severe conditions. Inspection should also focus on the vehicle's special parts like engine exhaust owing to the bad operational condition. They suffer much more corrosion and should be checked and serviced regularly.

Coatings of bare connectors. They are easy to be damaged and hence the electric connection performance would be influenced consequently. A small area of coating damage could be mended while connector with a large area of coating damage should be replaced with new one.

Rubbers and plastics. Rubber ring or cushion should be replaced with new one when they have declined elasticity or flaws which affect thier performance. The plastic or rubber sheath around an electric cable should avoid over bending. The paulin wrapping up cable beams should be painted even replaced when breakages happen.

\section{Summary}

Some corrosion control practices are implemented to improve the corrosion resistant for ground vehicles, which prove to be effective and economical. There are still some maintenance challenges due to anticipated corrosion in current technology or operational environment, so more and better ways should be developed from best practice lessons learned to support optimal corrosion control.

\section{Acknowledgement}

This research was financially supported by the GAD key program (Project Number:ZLA14065).

\section{References}

[1] B. Jegdić, S. Ristić, A. Alil, Corrosion processes, nature and composition of corrosion products on iron artefacts of weaponry, Scientific Technical Review, 61(2011)50-56.

[2] Greg Bock, Impact of corrosion on ground vehicles, field site operations. VSE Corporation, ADA533764, 2010.

[3] GD Davis, RA Ross, S Raghu, Coating health monitoring system for army ground vehicles, Corrosion Conference and Expo (CORROSION 2007).

[4] Antonio R. Mendoza, Francisco Corvo, Outdoor and indoor atmospheric corrosion of non-ferrous metals, Corrosion Science, 42 (2000) 1123-1147.

[5]P. Marcus, Corrosion mechanisms in theory and practice, third ed., Taylor \& Francis / CRC Press, 2011.

[6] Office of DoD Corrosion Policy and Oversight, Corrosion prevention and control planning guidebook for military systems and equipment, U.S. Department of Defense, 2014. 